\title{
eJRIEPS
}

Ejournal de la recherche sur l'intervention en éducation physique et sport

Numéro spécial 1 | 2018

Comment penser les articulations entre approches

didactiques et ergonomiques pour étudier les

pratiques d'enseignement et de formation?

\section{Une approche ergo-didactique des enseignements artistiques, en éducation musicale et arts plastiques}

An Ergo-Didactic approach of artistic teachings, in Musical Education and Visual Arts

Laurence Espinassy et Pascal Terrien

\section{OpenEdition}

Journals

Édition électronique

URL : http://journals.openedition.org/ejrieps/293

DOI : $10.4000 /$ ejrieps.293

ISSN : 2105-0821

Éditeur

ELLIADD

Référence électronique

Laurence Espinassy et Pascal Terrien, « Une approche ergo-didactique des enseignements artistiques, en éducation musicale et arts plastiques », eJRIEPS [En ligne], Numéro spécial 1 | 2018, mis en ligne le

01 avril 2018, consulté le 01 août 2019. URL : http://journals.openedition.org/ejrieps/293 ; DOI :

10.4000/ejrieps.293

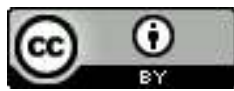

La revue eJRIEPS est mise à disposition selon les termes de la Creative Commons Attribution 4.0 International License. 
eJRIEPS Numéro Spécial n 1 Avril 2018

Une approche ergo-didactique des enseignements artistiques,

en éducation musicale et arts plastiques

Laurence Espinassy et Pascal Terrien

Aix Marseille Univ, ADEF, Marseille, France

Résumé

Notre étude porte sur des pratiques scolaires dont les productions plastiques et musicales se voient ou s'entendent immédiatement en classe. Pourtant, malgré cet aspect publiquement partageable, les enregistrements vidéo de séances de cours ne permettent pas au spectateur de déchiffrer comment les professionnels des enseignements artistiques conçoivent et réalisent leur travail, sur des bases qu'ils jugent efficaces, à la fois pour les élèves et pour eux-mêmes. Nous interrogeons d'une part, l'intérêt d'une approche ergo-didactique pour penser l'activité professorale dans les enseignements artistiques, et d'autre part, l'éventuel usage de ces enregistrements en formation professionnelle, pour aider à expliciter l'enchevêtrement des dimensions ergonomique et didactiques des multiples organisateurs de l'activité enseignante.

Mots clés: Activité enseignante - Arts plastiques - Éducation musicale - Analyse ergonomique et didactique

Nous proposons de mobiliser une double approche, didactique et ergonomique, pour analyser deux documents proposés comme ressource de formation, l'un en arts plastiques, l'autre en musique. Notre objectif est de lever en partie l'opacité de l'activité professionnelle des enseignants filmés par une analyse des séquences, et d'ouvrir plus généralement la réflexion sur l'utilisation de telles ressources en formation professionnelle dans ces deux disciplines qui ont des caractéristiques communes.

Leurs particularités résident, d'une part, dans leurs rapports à l'ampleur et la diversité de leurs champs de pratiques et de savoirs, systématiquement liés à l'expérience pratique et esthétique : les élèves étant mis systématiquement en situation de pratique plastique ou musicale en classe (Espinassy \&Terrien, 2017), ils éprouvent que : "la forme est en guerre avec le contenu, elle se bat avec lui, en triomphe, et c'est dans cette contradiction 


\section{eJRIEPS Numéro Spécial n¹ Avril 2018}

dialectique entre le contenu et la forme que se situe le sens psychologique de la réaction esthétique » (Vygotski, 1925/2005).

D'autre part, ces deux disciplines artistiques participent des situations professionnelles en éducation qui se caractérisent par un déficit de prescription: les programmes d'enseignement n'indiquent pas de tâches précises, et il n'existe pas de manuel scolaire, ou de "mode d'emploi " des programmes (hormis les documents d'accompagnement à leur mise en œuvre, mis en ligne sur Eduscol, que l'on peut considérer comme des prescriptions secondaires) ; «On est typiquement dans la situation où la prescription est infinie et la sous-prescription des moyens pour les atteindre est totale » (Daniellou, 2002).

La mobilisation d'une double approche, didactique et ergonomique, auprès de disciplines d'enseignement impliquant la pratique artistique au cœur de leur activité, voudrait contribuer à mieux cerner la manière dont les professionnels de l'éducation dans les domaines artistiques conçoivent et réalisent leur travail. Comment se transmettent des « compétences artistiques »? Autour de quelles activités ? Ces questions en soulèvent d'autres d'ordre méthodologique, car pour saisir « l'expérience subjective du travail », les approches cliniques se heurtent à une difficulté fondamentale liée au caractère incorporé, peu dicible et opaque de l'expérience professionnelle, d'autant plus quand elle s'intéresse à l'expérience artistique et esthétique.

Nos analyses des phénomènes d'apprentissage et d'enseignement s'intéressent aux multiples facettes et finalités du travail enseignant; elles visent à élucider le rôle que jouent les gestes de métier et leur imbrication avec les savoirs didactiques dans la recherche d'une efficacité pédagogique dans une situation particulière (Félix \& Saujat, 2008). Si les approches didactiques se focalisent sur les modalités de (co)construction des savoirs reliés aux pratiques culturelles qui les légitiment (Brière-Guenoun, 2017), les approches ergonomiques analysent l'activité réelle des sujets dans des tâches prescrites qu'ils redéfinissent.

Par une approche comparative entre didactique des arts plastiques et de l'éducation musicale, que l'on oppose souvent, nous cherchons à conceptualiser les rapports entre l'activité professorale, l'action didactique (définir, dévoluer, réguler, institutionnaliser) et les milieux dans lesquels ils se développent. Nous proposons d'interroger les tensions dynamiques de l'activité, entre action, connaissance, gestes et savoirs. Dans cet objectif, nous mobilisons nos champs de formation respectifs. 


\section{eJRIEPS Numéro Spécial n¹ Avril 2018}

Les cadres théoriques invoqués en didactique croisent les apports de la théorie des situations didactiques (Chevallard, 1985), notamment la notion de milieu didactique (Brousseau, 1998 ; Johsua \& Dupin 2001 ), les apports de la théorie de l'action conjointe en didactique avec les notions liées au triplet didactique et celles liés au jeu (Sensevy et Mercier, 2007).

Le cadre de l'analyse ergonomique de l'activité dans une approche historico-culturelle héritière de Vygotski et de Leontiev, considère que les résultats de l'activité humaine constituent des ressources ou des moyens d'action pour l'activité à venir.

Le croisement de ces orientations théoriques permet d'étudier les deux documents vidéos en questionnant la notion de milieu comme espace pluridimensionnel (Brousseau, 1998 ; Sensevy, 2007). Par «milieu», nous entendons aussi une construction chargée d'histoire, de culture, de « social » et de techniques. À la suite de Canguilhem (1947) et de Wallon (1954), nous considérons qu'un être vivant n'est pas simplement situé dans un environnement : il est en relation avec un milieu. Ce dernier n'est pas seulement une somme de données physico-chimiques : c'est un carrefour de significations vitales.

En classe, ce milieu est à la fois un artefact matériel et symbolique, constitué par des objets culturels, des signes ou des outils, mais comme le rappelle Brousseau (1998, p.63) : « un milieu sans intentions didactiques est manifestement insuffisant à induire chez l'élève toutes les connaissances culturelles que l'on souhaite qu'il acquière ».

Ce qui nous intéresse dans les situations d'enseignement, c'est la conception et l'organisation de «tout ce qui agit sur l'élève ou ce sur quoi il peut agir » (Brousseau, 1998, p. 32), et en approfondissant l'approche développée par Johsua et Félix (2002, p. 100), agissant comme une composante non négligeable du système didactique, qui impacte l'enseignant et l'apprenant. Plus que l'idée « d'un milieu à trou » prenant en compte l'ignorance de l'élève (Johsua \& Félix, 2002), il s'agit de voir dans ces dispositifs des milieux qui suscitent, qui stimulent, qui développent l'apprentissage en s'appuyant sur les caractéristiques de l'activité installée par la prescription descendante de l'enseignant et celles remontantes des élèves (Daniellou, 2002).

\section{Contextes et nature des supports des séances analysées}

1.1. En arts plastiques

\subsubsection{Contexte}

Notre analyse porte sur un document vidéo produit par Canope Nancy-Metz en 2016, à 
eJRIEPS Numéro Spécial n¹ Avril 2018

l'initiative de Sophie Renaudin, IA-IPR d'arts plastiques. II est indiqué comme ressource d'accompagnement des programmes du cycle 3, accessible par le portail Eduscol soit dans son intégralité, soit par segments renvoyant à des enjeux pédagogiques ou des temporalité particulières des apprentissages, indiqués par des titres et sous-titres. II s'agit d'une séance d'arts plastiques où l'on donne à voir le travail mené par les élèves sous la conduite d'un professeur des écoles (PE) et les éclairages offerts par des entretiens menés avec l'enseignant, inclus au document vidéo. II s'agit d'une classe de CM1, en début de cycle 3, qui, depuis la mise en œuvre de la loi de Refondation de l'École (B.O. 8/07/2013) et de la Réforme du collège (J.O. du 20/05/2015), regroupe les cours moyens $1^{\text {ère }}$ et $2^{\text {me }}$ année, ainsi que la sixième. Ce cycle a donc pour particularité de s'inscrire à la transition entre école primaire et collège.

Ce document n'est donc pas directement issu de nos matériaux de recherche ; filmé par une IPR et destiné aux PE, il constitue néanmoins, pour l'analyste du travail enseignant et spécialiste des AP que nous sommes, un support d'étude fort intéressant pour croiser les cadres théoriques et les outils de l'ergonomie de l'activité et de la didactique des arts plastiques. En effet, proposé en tant que ressource de métier (très rares sous forme filmique en $\mathrm{AP}$ ) à destination des enseignants du cycle 3 , rien n'indique que des $\mathrm{PE}$ non spécialistes de la discipline, mais qui souhaitent s'y intéresser par le biais de ce document vidéo, puissent déceler les multiples facteurs orientant l'activité enseignante ici donnée à voir. Cette hypothèse est confirmée par la présentation de cette ressource lors d'un plan de formation: même si le PE enregistré donne des éclairages sur ses intentions didactiques dans ses commentaires, et que les titres des chapitres orientent la lecture de la situation, il n'est pas garanti que des tiers extérieurs puissent s'emparer des ressorts cachés de l'action visible. Conscients que nous n'avons pas mis en œuvre de méthodologies indirectes, comme c'est habituellement le cas dans nos recherches (Clot \& Faïta 2000, Faïta \& Saujat 2010), et que, dans ce type de document, le discours de cet enseignant est forcément adressé de façon indirecte à des tiers qui souhaitent se former, nous avons pris le parti de nous y arrêter. L'ensemble des échanges langagiers en classe, ainsi que les commentaires de l'enseignant à propos de son activité et de celle de la classe, ont été transcrits. C'est ce matériau verbal (retrouvé ci-après entre guillemets et en italique, en suivant la chronologie de la séance), ainsi que l'observation de ce qui se fait en classe qui servent de base à notre analyse.

\subsubsection{La situation d'enseignement}


eJRIEPS Numéro Spécial n¹ Avril 2018

La salle de classe, un carré-rectangle, est aménagée en trois tablées longitudinales de 6, pour un effectif de 18 élèves ; l'un des petits côtés est occupé par le tableau et d'autres affichages de grande taille; de l'autre côté on trouve un grand écran d'ordinateur sur une table dans un espace suffisamment dégagé pour permettre autour le regroupement des élèves. Partout, des étagères et des panneaux rendent visibles les travaux déjà accomplis.

Dans l'extrait filmique 1, intitulé « Du contexte à la mise en œuvre », la situation de classe est ainsi résumée par le PE dans son commentaire post-séance : “il s'agit d'une projection dans un monde imaginaire que les élèves ont inventé ; on est en 2116 , et il nous faut trouver des solutions possibles aux problèmes environnementaux qui se poseront, et qui se posent déjà ». Dans le cadre de l'enseignement polyvalent de l'école primaire, il s'agit d'un projet transdisciplinaire qui allie écriture et APL, et qui s'inscrit dans le cadre de l'Éducation au Développement Durable (MEN, 2012-2015).

\subsection{En musique}

\subsubsection{Le contexte}

Le document vidéo retenu est extrait d'un début de cours d'initiation à la contrebasse filmé par l'enseignante dans un conservatoire à rayonnement régional de musique, de danse et de théâtre, en 2014. Considérant que la formation des professeurs de musique intervenant au sein des écoles de musique et conservatoires s'est peu à peu développée depuis la mise en place des premiers centres de formation des enseignants de musique (CEFEDEM), peu de recherches ont été à ce jour réalisées sur les pratiques enseignantes de ces professeurs, et encore moins sur l'analyse didactique des activités enseignantes (Regnard \& Cramer, 2003 ; Terrien, 2011, 2015). Ce document fait partie des matériaux à partir desquels nous élaborons nos recherches sur milieu didactique d'une situation de cours (Brousseau, 1998 ; Sensevy \& Mercier, 2007 ; Terrien, 2015) et nous permet de mener, dans le contexte de nos travaux, des analyses sur le travail des musiciens-enseignants quelle que soit la discipline pratique ou théorique dispensée dans un conservatoire.

Dans le cadre de l'éveil à la musique, il est proposé aux enfants des écoles primaires de cette ville n'ayant pas encore sept ans, et inscrits au conservatoire dans le «parcours découverte », des cours d'initiation aux instruments sur l'ensemble de l'année scolaire. Les élèves choisissent, lors de cette première année, avec leur professeur d'éveil musical sept instruments parmi ceux enseignés au conservatoire, dont trois leur sont imposés pour qu'ils puissent découvrir ceux vers lesquels ils n'iraient pas spontanément. 
eJRIEPS Numéro Spécial n¹ Avril 2018

Les cours sont organisés sous forme d'atelier et par groupe de trois élèves. Chaque élève dispose de trois séances de 30 minutes avec le professeur du conservatoire qui peuvent être organisées en cours particulier et en cours collectifs.

\subsubsection{La situation d'enseignement}

II s'agit d'un premier cours de contrebasse s'inscrivant dans cette formation d'éveil aux instruments de musique. L'élève que nous appellerons Camille est âgée de 6 ans et demi, élève en cours préparatoire à l'école, elle n'a jamais pratiqué l'instrument. La séquence filmée de 4'44" montre son premier contact avec la contrebasse. Dans le cadre de ce cours et vu l'âge des enfants, il s'agit d'une $8^{\text {ème }}$ de contrebasse, une des tailles les plus petites de l'instrument.

\section{Analyse des séquences}

2.1. Analyse et résultats en arts plastiques

2.1.1. Intentionnalité didactique

Afin de réactiver la mémoire collective de travail, les élèves sont regroupés autour de l'écran, et l'un d'eux lit de façon très solennelle le « Communiqué du Grand Conseil Planétaire » qu'ils ont rédigé ensemble auparavant. Dans leur scénario, les élèves ont imaginé que dans cent ans, les dérèglements climatiques et la surpopulation feront qu'en 2116 : « on va diminuer de taille », et ils la déterminent à « environ $17 \mathrm{~cm}$ ». En termes d'engagement collectif, il s'agit de construire la nouvelle situation de travail à partir de cette base commune ; ce sont les élèves qui ont donné ce point de départ, et ce n'est pas le PE qui l'impose. II explique à la classe : « Aujourd'hui en $\boldsymbol{A P}$, on va imaginer comment pourrait être notre nouvelle maison ».

Le PE enchaîne : “À votre avis, qu'est-ce que je pourrai vous proposer comme matériau pour la réaliser ? ». Cette question laisse aux élèves le temps de spéculer sur les diverses potentialités qu'offrent certains matériaux de construction qu'ils connaissent déjà ; suite à cette ouverture de possibles, il est annoncé que c'est l'argile qui serait utilisée. Dans son commentaire de la séance, l'enseignant explique ce choix : "Nous avons déjà travaillé l'argile dans une séance qui a fait l'expérience des qualités du matériau. On avait abordé les notions d'intérieur et d'extérieur, mais ce n'était pas apparu dans une problématique d'architecture. Là, je voulais les mettre dans une situation d'architectes en herbe, et entrer dans cette problématique de l'espace intérieur habitable ». Rappelons-nous que cet enseignant n'est pas un spécialiste de l'enseignement des AP. 


\section{eJRIEPS Numéro Spécial n¹ Avril 2018}

Pour autant, il manifeste une fine connaissance des objectifs spécifiques en AP énoncés dans les programmes du cycle 3 , qui pourraient être tout à fait approfondis ensuite en cycle 4. Notons que sa réflexion pédagogique s'inscrit dans une progressivité liée autant à l'expérience du matériau, qu'à la complexité des questions plastiques de l'architecture. II ne s'agit donc pas simplement de fabriquer de petites maisons pour de petits bonhommes ... : l'intention didactique et les liens aux savoirs disciplinaires sont bien présents.

2.1.2. Des prescriptions aux réalités du contexte : ajustements et compromis opératoires

À la suite, dans son commentaire, le professeur précise : "Un des écueils quand on travaille l'argile avec les enfants, c'est de proposer en même temps l'argile et l'eau. Ici, on a une préoccupation de solidité liée à l'architecture et si l'argile ramolli avec l'eau, on aurait des difficultés insurmontables pour les enfants". Fort de son expérience, et vraisemblablement de difficultés préalablement rencontrées (car elles peuvent vite devenir insurmontables aussi pour le PE...), il anticipe les problèmes à venir, à la fois pour les élèves et pour lui-même. Considérant les contraintes de faisabilité en regard des objectifs d'apprentissage et du matériau choisi, il adapte la situation aux réalités du contexte et au niveau des élèves en effectuant un choix: supprimer l'eau, c'est restreindre volontairement le champ des gestes techniques pour rester concentré sur les objectifs à atteindre ; c'est donner moins pour espérer produire plus et mieux.

\subsubsection{Un milieu aménagé pour apprendre}

Le PE a préparé un bloc d'argile, d'environ $10 \mathrm{~cm}^{3}$ pour chacun des élèves ; ces cubes reposent individuellement sur un carreau de faïence, et sont disposés en 3 rangées de 6 sur une table. Chaque élève viendra chercher le sien. Ce dispositif peut paraître anodin ; malgré cette apparente simplicité, il recèle de nombreux ressorts didactiques. 




Document 1 : Les cubes d'argile à disposition des élèves en début de séance

Il se trouve que le cube et la base carrée sont les fondements de l'architecture, qui d'emblée offrent ici une entrée en matière, à tous les sens du terme, aux savoirs disciplinaires et aux objectifs visés, tant du point de vue de la pratique plastique, que des références au champ de l'histoire des arts. II serait facile de les repérer autant dans l'histoire ancienne (mastaba, temples...) que dans des constructions contemporaines (le Mucem de Riciotti à Marseille, par exemple). De plus, posés ainsi côte à côte et alignés, cet ensemble de blocs ressemble déjà à une petite ville (ce qui a une importance dans la suite du projet). II est également possible de rapprocher l'ensemble bloc/base aux fondements de la sculpture et au dispositif de présentation matérialisé par le socle. Ainsi, cette manière d'agencer le milieu matériel, est déjà pour cet enseignant une manière implicite de définir les objets didactiques qui seront travaillés (architecture et espace intérieur habitable, liés à l'expérience des qualités du matériau, B.O. Spécial $N^{\circ} 11$, du 26/11/2015), et crée une ouverture vers l'Éducation Artistique et Culturelle (B.O. 01/07/2015) et l'histoire des arts.

D'un point de vue pragmatique, c'est manipulable par des mains d'enfants, à leur échelle ; l'argile ne colle pas au carreau, les tables restent propres (puisqu'il n'y a pas d'eau), en outre, c'est aussi facile à déplacer et pratique à ranger pour le professeur. Les dimensions du bloc et du carreau, similaires pour chacun, définissent la topogenèse de l'action à venir. 


\section{eJRIEPS Numéro Spécial n¹ Avril 2018}

Mais au-delà de ces dimensions didactiques cachées, ce n'est qu'une fois qu'ils seront confrontés à leur cube d'argile que les élèves vont découvrir le problème auquel ils sont confrontés et dont ils sont eux-mêmes en partie responsables : pour qu'un habitant de 17 $\mathrm{cm}$ puisse entrer dans une maison, même très modeste, qui lui soit adaptée, un cube de $10 \mathrm{~cm}^{3}$ est manifestement trop petit. En terme de dévolution de la tâche, il va donc falloir l'élever (pratiquement au double), le modifier, le creuser... pour rendre son espace intérieur habitable, dans le temps limité de la séance.

Aucune des dimensions (problématique, didactique, fonctionnelle, procédurale, chronogénétique) décrites jusqu'alors et sous-jacentes à ce dispositif d'enseignement n'ont été annoncées aux élèves, pas plus qu'aux usagers de ces ressources professionnelles.

\subsubsection{Pour conclure à propos de la situation en arts plastiques}

En AP, l'acquisition des connaissances et des compétences est construite, principalement à partir des questions que fait rencontrer la pratique. Le travail de l'enseignant consiste donc à retravailler les prescriptions officielles, pour à son tour, prescrire des tâches aux élèves en vue d'une réalisation plastique en classe. C'est-à-dire qu'il doit concevoir et faire fonctionner un milieu de travail (permettant la production plastique) et un milieu didactique permettant l'atteinte de certains objectifs d'apprentissages des programmes d'enseignement. Ainsi, la conception des situations d'enseignement en AP, nécessite la conjonction de la double visée ergonomique et didactique ; elle doit permettre la mise en activité collective des élèves, l'apprentissage et le développement personnels de chacun, jusqu'à la réalisation plastique en situation. L'aspect visible et comparable des productions permet l'institutionnalisation des propositions des élèves en regard des savoirs et compétences visées.

Nous qualifions ces milieux d'enseignement « d'ergo-didactiques", car leur conception tente d'évaluer et d'anticiper "l'espace des formes possibles de l'activité future " (Daniellou, 1992), celle de ses élèves mais aussi cette du professeur en réponse à la leur, tout en atteignant les objectifs des programmes. Si l'on considère avec Wisner, que travailler consiste moins à appliquer les prescriptions qu'à " vaincre des difficultés " (1995), notre entrée permet d'entrevoir le métier, " simultanément, comme problème et comme solution " (Clot, 2008), dans l'enchevêtrement des enjeux didactiques et des enjeux ergonomiques. Du point de vue de l'activité professorale, la situation ici étudiée reflète, à la fois, d'un souci d'efficacité et de recherche de pertinence didactique, autant que d'efficience et d'économie. Sous l'apparence simplicité du dispositif minimal des 


\section{eJRIEPS Numéro Spécial n¹ Avril 2018}

cubes d'argile se cache une grande complexité de conception.

2.2. Analyse et résultats en musique

2.2.1. Intentionnalité didactique

L'action se déroule dans une salle de cours dédiée à la formation musicale (solfège), d'où la présence de tables et de chaises à l'image. La professeure de contrebasse a dégagé un espace qui lui permet de poser l'instrument sur son éclisse (présentant ainsi à l'élève la face de la contrebasse avec le chevalet et les cordes) et qui autorise l'élève à évoluer sans être contraint par l'environnement matériel. L'archet de l'instrument est posé sur une table qui est derrière l'instrument et qui, de ce fait, se trouve à la droite de l'élève lorsque celle-ci souhaitera le prendre.

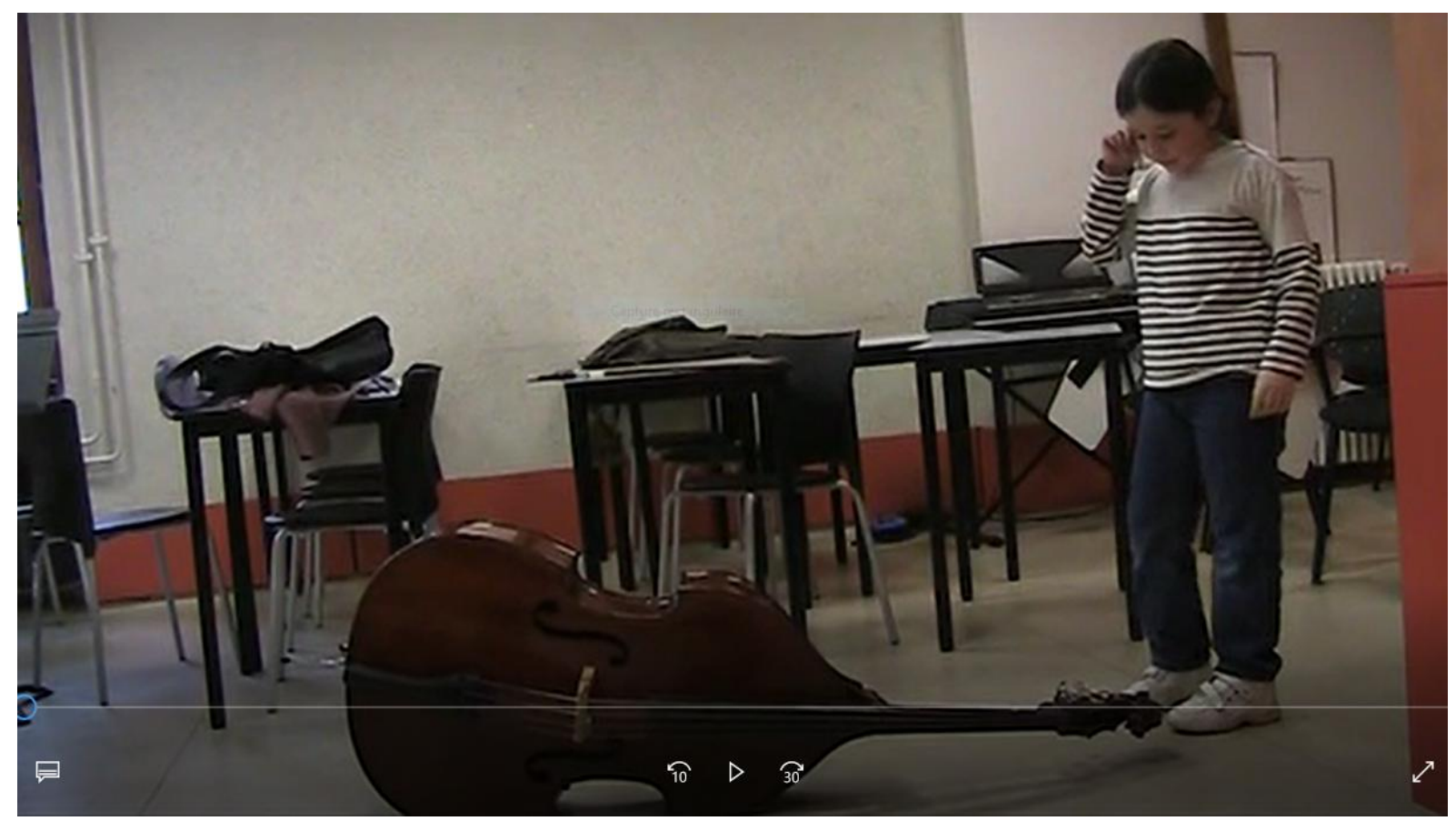

Document 2 : les premiers gestes vers la contrebasse

L'objectif de la professeure est de faciliter le premier contact avec l'instrument et de sécuriser sa prise en main. Conformément aux directives du Schéma national d'orientation pédagogique de 2008, elle répond ainsi à « La mission première des établissements étant de former des amateurs, les établissements veilleront à favoriser les liens avec la pratique en amateur existant à l'intérieur ou à l'extérieur du conservatoire, afin qu'un grand nombre d'élèves poursuivent leur pratique artistique au-delà des enseignements du conservatoire. "(SNOP, 2008, 7). Elle inscrit donc ces trois cours d'éveil à la contrebasse dans une sensibilisation à « une conscience contemporaine du phénomène sonore et musical » (Ibid.) en installant un environnement didactique qui 


\section{eJRIEPS Numéro Spécial n¹ Avril 2018}

permet aux élèves de découvrir les ressources sonores de la contrebasse. D'autre part, cette professeure déclare "que la première année, [elle] met en place beaucoup de pratiques d'ensemble, d'audition, [...] de recherche sur le son de l'instrument pour sensibiliser les élèves au rapport entre la tenue de l'instrument, de l'archet, et la production sonore. "

2.2.2. Des prescriptions aux réalités du contexte : ajustements et compromis opératoires

La professeure organise l'espace de la classe pour que les élèves découvrent l'instrument en toute sécurité, et puissent le manipuler sans être gênés par les objets, mais en même temps elle indique qu'il faut : "un territoire un peu petit, pas trop grand, car cela, ça les impressionne quand la salle est trop grande qu'il n'y a rien dedans. Je fais juste de la place pour qu'il y ait un territoire de jeu ». Interrogée sur le placement de l'instrument, en posant le devant sous les yeux de l'élève, elle ajoute " si, tout de même, effectivement, je la pose [la contrebasse] de façon à ce qu'elle voit les cordes, le chevalet [...], et l'archet est posé sur la table derrière la contrebasse de façon à ce qu'elle voit l'archet, l'archet et la contrebasse [...], cela doit lui permettre de tourner autour. " Dans le même ordre d'idée, la professeure nous indique : " $d u$ coup, il n'y a pas d'objet de distraction parce que si je mets une boite d'archet, ils se demandent ce que c'est, du coup ils ne sont plus centrés du tout sur l'instrument, donc là, il y a juste leurs affaires qui sont posées sur les tables. " (Entretien d'autoconfrontation simple avec la professeure). Dès lors, lorsque les enfants entrent dans la classe, ils voient l'instrument et l'archet posés sur la table, et comme ils ont été sensibilisés par le cours d'éveil aux instruments, ils ont déjà vu sous forme d'image ou de vidéo des instruments à cordes. Ils possèdent donc des notions sur ces instruments, notamment qu'ils se jouent avec un archet.

La professeure cherche à ce que ces élèves prennent sans appréhension l'instrument, qu'ils produisent très rapidement des sons, soit pizzicato (en pinçant les cordes avec les doigts), soit arco (en frottant avec l'archet). Elle cherche à profiter du manque d'inhibition propre à l'âge de ces enfants "parce qu'après ils sont moins anarchistes, ils hésitent plus... ». En même temps, le dispositif imaginé amène l'élève à prendre la contrebasse à deux mains sur le manche, ce qui porte la professeure à nous faire une remarque sur la prise de l'instrument : " si elle avait eu son premier cours de contrebasse elle ne l'aurait pas prise comme ça, parce que lors des premiers cours d'instrument, la première chose que je fais c'est de leur apprendre à prendre l'instrument... » et elle nous explique alors la manière la plus efficace de prendre la contrebasse sans risque de la voir échapper des 


\section{eJRIEPS Numéro Spécial n¹ Avril 2018}

mains de l'élève et de tomber.

2.2.3. Un milieu aménagé pour apprendre

Dans cette vidéo, on observe la manière dont Camille va prendre en main la contrebasse. Lorsqu'elle s'avance vers l'instrument, elle le regarde et en même temps remarque l'archet posé sur la table (0'-0'15'). Elle fait le tour le d'instrument et regarde cette fois le dos de la contrebasse et l'archet posé sur la table, et semble se demander comment prendre les deux objets ensemble. C'est alors qu'elle se tourne vers la professeure en quête d'une approbation :

0'15 E : on commence...

0'16 P : et oui j'te laisse (court silence), allez on dit que tu as 10 minutes un quart d'heure, euh... je te laisse, euh

0'20 E : 10 minutes un quart d'heure...

0'21 P : ouais, ah, tu fais comme tu veux.

0'24 E : bon ben... (et elle se penche pour prendre l'instrument)

Cet échange verbal introduit l'activité de prise en main de l'instrument par Camille et établit les bases d'un contrat didactique. Ce rappel à la prescription « découvre l'instrument en le prenant et en jouant » par la professeure est relayé par le rappel du temps dont Camille dispose pour réaliser cette tâche, en même temps le ton de la professeure est suffisamment bienveillant pour que l'enfant s'autorise l'activité.

En termes de topo et chronogenèses, la situation de travail est circonscrite dans le temps et l'espace par l'enseignante, comme elle nous l'explique ci-dessus, et elle encourage son élève à poursuivre ses activités de découverte. L'enseignante adapte les contraintes de l'environnement, de la prescription, de l'objet (une contrebasse n'a pas les dimensions d'une flûte à bec), à celle liées à l'âge de l'enfant, sa taille, sa force, ses savoir-faire et ses connaissances. En restreignant également le champ des activités potentielles que l'enfant pourrait accomplir, elle lui permet aussi de développer celles qui sont les plus immédiatement utiles à l'exploration de l'instrument et pour résoudre la tâche. D'autre part, vu l'âge des enfants (moins de sept ans), la professeure met à leur disposition une $8^{\text {ième }}$ de contrebasse. La dimension de l'instrument devrait leur permettre de s'en saisir facilement, mais pourtant une petite élève laissera l'instrument au sol pour en découvrir le potentiel sonore. Les dimensions de l'instrument adaptées à l'âge des élèves leur permettent de prendre des risques dans la réalisation de leur activité, tout en économisant leur effort ; car, les petits élèves, tels que Camille, découvrent vite les difficultés et la 


\section{eJRIEPS Numéro Spécial n¹ Avril 2018}

souffrance que peut apporter le jeu instrumental, et il est nécessaire de créer un rapport supportable entre l'instrument et leur corps.

Ainsi, sous son apparente simplicité, l'organisation de ce milieu dissimule de nombreux contrats didactiques implicites entre la professeure et son élève dont les dimensions ne sont pas forcément visibles pour un professeur novice.

\subsubsection{Pour conclure à propos de la situation en musique}

En musique, l'apprentissage instrumental passe par l'acquisition de savoir-faire fondés sur la pratique. Traditionnellement, l'apprentissage instrumental est dirigé par le professeur qui donne des prescriptions à son élève. Celui-ci réalise l'activité sans forcément se questionner sur le sens où la finalité du geste. Pour la réalisation de cette vidéo, l'enseignante a conçu une situation didactique où le principe de dévolution doit lui permettre de cerner les savoir-faire et compétences déjà acquis par son élève afin de pouvoir l'aider dans le développement de son activité d'apprentissage. II apparaît que la seule prescription de prendre en main l'instrument et d'explorer les modes de jeux cachent des tâches implicites qui devront être réalisées à l'initiative d'activités faites par l'élève. Tel est la conception du milieu de travail qui est aussi un milieu didactique mettant l'élève en activité.

La notion de milieu d'enseignement « ergo-didactique » est ici reprise, parce qu'il permet au professeur et à l'élève de faire un bilan sur ce qui a été fait, ce qui n'a pas été réalisé et ce qui est possible de faire en dépliant les possibles de l'activité enseignante et apprenante (Félix, Saujat, Combes, 2012). Cette situation offre donc au professeur des perspectives de développement de l'activité (Clot, 2008) en corrélation avec les capacités singulières de l'élève qu'il a en cours. Cette situation de cours cache plus d'implicite que ce qu'elle ne donne à voir, car pour en expliciter les déroulements, il est nécessaire de comprendre la complexité que recouvre la découverte et la première prise en main d'un instrument de musique.

\section{Discussion : entre recherche et formation}

Les deux supports vidéo présentés ici proviennent de sources différentes et sont proposés comme ressources de formation. Si les enseignements artistiques visent le développement de l'adaptabilité de l'enfant, de son autonomie, de sa créativité, alors: «Chaque fois qu'on explique quelque chose à un enfant, on l'empêche de l'inventer » (Piaget in Bringuier, 1977). De fait, les situations d'enseignement que nous avons décrites 


\section{eJRIEPS Numéro Spécial n¹ Avril 2018}

démythifient la nécessité d'enseignements ou d'éducation préalables à tout apprentissage et interrogent la place et le statut de l'enseignant prescripteur et transmetteurs de savoirs, dans une logique «Top-Down» (qui reste encore aujourd'hui très forte, notamment dans l'enseignement de la musique). En tant que formé, comment apprendre à concevoir ces dispositifs d'enseignement qui n'ont pas pour vocation à faire de «l'Art », et dont l'objet est de développer chez l'élève des aptitudes créatives et des questionnements sur les démarches artistiques et sur les œuvres d'art?

En adoptant le point de vue du formateur, nous imaginons quelques usages éventuels de ces supports documentaires.

\subsection{Une formation à visée explicatrice}

Le document concernant les arts plastiques donne à voir et à analyser le déroulement d'une séance de pratique plastique déclinant les différentes phases de travail des élèves partant de la prescription formulée par le PE, et assorties de ses commentaires successifs. Dans ce cas, en adoptant une posture d'observateur, l'objectif de la formation sera d'outiller les enseignants débutants afin qu'ils puissent décrypter la situation didactique, relever la richesse des échanges entre le PE et ses élèves, et examiner les activités de chacun pour réaliser une première analyse des actions menées par les uns et les autres. Ces extraits vidéos peuvent servir de support pour comprendre l'articulation entre action didactique et activité professorale au sens large, pour réfléchir sur l'organisation de l'espace, sur le maniement du matériel, sur les liens entre prescription et réel de l'activité, entre conception et mise en pratique, etc., non seulement du point de vue de la pertinence des objectifs spécifiques à la didactique des arts plastiques, mais audelà, du point de vue de la polyvalence disciplinaire des PE, et des différents aspects génériques de l'agir professionnel de l'enseignant. Le formateur peut aussi intéresser les PE débutants au caractère dynamique de l'activité (Theureau, 2006) en leur apprenant à discerner « celle qui s'ancre dans des expériences passées proches ou plus lointaines et qu'elle préfigure des actions futures »(Leblanc, Ria, Veyrunes, 2013).

Pour résumer, ce document vidéo est potentiellement un support d'analyse du travail, autant celui des élèves que du professeur, pour tenter d'objectiver les actions des uns et des autres et en s'appuyant sur les commentaires livrés a posteriori par l'enseignant. Mais, restant déconnectée de l'activité de travail réel des formés, rien ne dit qu'un tel type de formation puisse constituer des ressources pour leur action future, en situations d'enseignement.

\subsection{Une formation à visée transformatrice}




\section{eJRIEPS Numéro Spécial n¹ Avril 2018}

La scène filmée sur la découverte de la contrebasse par une élève débutante est d'une autre nature puisqu'elle s'adresse non plus à des professeurs des écoles mais à des professeurs de musique dont les missions et les tâches s'inscrivent dans un environnement professionnel différent. Dans la situation proposée, l'environnement didactique est délimité a priori par la professeure de musique et, comme nous l'avons décrit ci-dessus, l'élève n'a pas d'autre contrainte que celle d'agir pour produire les premiers sons sur l'instrument.

Du point de vue du formateur, cette vidéo réalisée par l'enseignant de musique s'inscrit dans le registre d'un processus de formation à visée transformatrice. En effet, lors d'un entretien d'autoconfrontation simple réalisé avec l'enseignante, elle va déplier l'activité de l'élève et par voie de conséquence la sienne ; elle va ainsi identifier des tâches implicites liées à la situation: celles qu'elle choisit de prescrire afin qu'elles offrent un panel d'activités possibles pour l'élève. Cette méthodologie indirecte de l'analyse de l'activité enseignante, telle que le cadre des autoconfrontations (Odonne, Rey, Briante, 1981 ; Clot, Faïta, 2000) provoque une activité réflexive de l'enseignante sur son dispositif didactique.

Dans une situation de formation de professeurs de musique cette vidéo permet au formateur d'accompagner les futurs professeurs dans une démarche réflexive (Clot, Faïta, Fernandez, Scheller, 2000) en les formant à l'analyse clinique et didactique de situations d'enseignement.

Enfin, la reprise de cet entretien par le chercheur permettra de mettre en relation l'analyse du milieu didactique (Johsua, Félix, 2002 ; Terrien, 2015), les propos de la professeure observée et ceux des professeurs en formation pour comprendre ce qui se joue dans le temps de la formation. Les résultats de cette recherche permettent d'imaginer d'autres dispositifs didactiques tant pour les élèves que pour la formation des enseignants.

\subsection{Vers une formation ergo-didactique?}

Ces deux vidéos présentent des dispositifs d'enseignement où les variables didactiques pédagogiques et organisationnelles semblent maîtrisées par les deux enseignants filmés. Mais, même si ces images recèlent un potentiel formatif, elles ne donnent à voir que l'aspect lisse du travail, sans qu'aucune des difficultés du métier n'y apparaisse, hormis dans quelques propos des enseignants concernés (par exemple, en AP : « ne pas donner d'eau avec de l'argile »; en Mus : « délimiter un territoire ni trop grand, ni trop petit... »). Comment faire comprendre à des professeurs en formation que c'est l'ensemble des organisateurs, autant didactiques qu'ergonomiques, de ces dispositifs d'enseignement, qui permet aux élèves d'évoluer dans un espace délimité et sécurisé ? Comment leur faire 


\section{eJRIEPS Numéro Spécial n¹ Avril 2018}

apprécier que le milieu pluridimensionnel conçu par l'enseignant, aide les élèves à s'autoriser des gestes créatifs ?

À nos yeux, l'analyse d'une situation d'enseignement dans une visée de formation des enseignants, doit être pensée d'un point de vue didactique, mais aussi comme une situation de travail.

L'enjeu de la formation est de créer un milieu dialogique entre les usagers de ces ressources et les milieux de travail et de recherche, pour ouvrir des zones potentielles d'apprentissage et de développement professionnels aux enseignants, (qu'ils soient débutants, expérimentés ou formateurs), afin qu'ils questionnent en retour l'efficacité de leurs propres dispositifs didactiques.

\section{Conclusion}

Les analyses de chacune des situations de cours, en musique et en arts plastiques, montrent la conception de ce que nous qualifierons de « milieux ergo-didactiques », que l'on peut tout autant considérer du point de vue du triplet didactique, mésogenèse, chronogenèse, topogenèse, que dans leurs rapports dynamiques entre les buts, les moyens et le sens de l'activité des acteurs (Leontiev, 1984). Pour autant, la complexité des organisateurs de ces situations d'enseignement échappe souvent au regard extérieur qui ne parvient généralement pas à identifier les ressorts cachés qui lient la tâche visible et l'activité réelle qu'elle suppose (Félix, Amigues, Espinassy, 2014 ; Develay, 1997 ; Clot, 2008).

Dans ces situations d'enseignement, la mise en relation des outils, des matériaux et de l'espace à disposition des élèves crée un milieu de travail individuel et collectif dynamique et efficace, dans l'enseignement de disciplines où il est impératif de faire faire pour faire apprendre. Dans les cas ici décrits, l'organisation artéfactuelle et sociale de ces salles de classe constitue un milieu spécifique qui offre les conditions nécessaires à l'appropriation des contraintes de réalisation transformées en outils pour agir. Cette organisation crée un système de contraintes et de ressources dans lequel vont évoluer les élèves pour répondre aux prescriptions posées par les professeurs (Espinassy, 2016 ; Espinassy, Saujat, 2016 ; Terrien, 2015).

Ces milieux ergo-didactiques sont emblématiques de la nécessité pour l'enseignant de concevoir une tâche destinée aux élèves comme un instrument bi-face « à la disposition d'acteurs engagés dans une collaboration asymétrique mais à visée partagée » (Vérillon, 
eJRIEPS Numéro Spécial n¹ Avril 2018

2005, p. 4). Ces situations sont simultanément un moyen de prendre la classe et d'en assurer le pilotage en fournissant aux élèves un sujet qui finalise et organise leur travail, et un objet de conception didactique qui devient pour eux un processus d'appropriation des façons de dire, faire et penser, tout en facilitant la circulation des savoirs qui fondent l'expérience artistique.

L'intérêt de ces deux vidéos dans une approche ergo-didactique, c'est que leur analyse plurielle et multifactorielle provoque la réflexion tant du point de vue de l'activité professorale, de l'ingénierie didactique, que de celui de l'apprentissage des élèves. De ce point de vue, nous rejoignons les préoccupations qui animent les chercheurs en didactique professionnelle : analyser l'activité pour « apprendre des situations professionnelles » et aider au développement des compétences, ici, pour le travail enseignant (Pastré, 2011, Mayen 1999, 2012).

\section{Bibliographie}

Brière-Guenoun, F. (2017). Instruire les gestes didactiques de métier. Quelles perspectives pour la formation des enseignants ? Presses Universitaires de Rennes.

Bringuier, J.-C. (1977). Conversations libres avec Jean Piaget. Paris : Robert Lafont.

Brousseau, G. (1998). Théorie des situations didactiques. Textes rassemblés et préparés par Balacheff, N., Cooper, M., Sutherland, R., Garfield. V. Grenoble : La Pensée Sauvage.

Canguilhem, G. (1947). Milieux et normes de l'homme au travail. (Cahier internationaux de sociologie, $\left.N^{\circ} 3\right)$. Paris : PUF.

Chevallard, Y. (1985). La transposition didactique: du savoir savant au savoir enseigné. Grenoble : La Pensée Sauvage.

Clot, Y. (2008). Travail et pouvoir d'agir. Paris : PUF.

Clot, Y., Faïta, D. (2000). Genre et style en analyse du travail. Travailler, 4, 7-42.

Clot, Y., Faïta, D., Fernandez, G., Scheller, L. (2001). Entretiens en autoconfrontation croisée: une méthode en clinique de l'activité. Clinique de l'activité et pouvoir d'agir. Education Permanente. $\mathrm{n} \cong$ 146/2001-1, 17-25, Genève.

Daniellou, F. (2002). Le travail des prescriptions, Conférence inaugurale du XXXVII Congrès de la SELF, Aix-en-Provence. En ligne : [http://ergonomie-self.org/wp- 


\section{eJRIEPS Numéro Spécial n¹ Avril 2018}

content/uploads/2016/01/congres-self-2002-aix-daniellou-travail-prescriptions.pdf], consulté le 29/08/2017.

Daniellou, F. (1992). Le statut de la pratique et des connaissances dans l'intervention ergonomique de conception. Note de synthèse pour l'Habilitation à Diriger des Recherches. Université de Toulouse-Le Mirail.

Develay, M. (1997). Savoirs scolaires et didactiques : une encyclopédie pour aujourd'hui. Paris : E.S.F.

Espinassy, L. (2016). Inciter à l'étonnement. D'une forme langagière à l'enseignement des arts plastiques. Enseigner la littérature en dialogue avec les arts. Confrontations, échanges et articulations entre didactique de la littérature et didactiques des arts. $17^{\mathrm{es}}$ rencontres des chercheurs en didactique de la littérature. IFE, ENS. Lyon, 1-3 juin. http://rdidlit17.hypotheses.org/espinassy

Espinassy, L., Terrien, P. (2017). Professeur débutant dans les disciplines artistiques : de l'expérience artistique du professeur à celles des élèves. In Boudinet, G. et Sanchez-lborra C. (Ed.), Expérience esthétique et savoirs artistiques (pp. 107118). Paris : L'Harmattan. Collection : Arts, transversalité, éducation.

Espinassy, L., Saujat, F. (2016). Enseigner les arts plastiques : organiser le travail des élèves pour tenir ensemble les dimensions didactiques, ergonomiques et créatives de l'activité des élèves et du professeur. $51^{\text {me }}$ Congrès de la SELF. Symposium : Pour une ergonomie de l'activité des professionnels de l'éducation et de la formation. 10 ans de recherches de l'équipe ERGAPE*: Apports et perspectives pour la recherche et l'action en ergonomie (Coord. L. Espinassy). Marseille, 21-23/09/16. https://hal.archives-ouvertes.fr/hal-01487490

Faïta D., Saujat, F. (2010). Développer l'activité des enseignants pour comprendre et transformer leur travail : un cadre théorique et méthodologique. In Yvon, F. \& Saussez F. (Eds), Des outils théoriques et méthodologiques pour l'intervention et la formation (pp.41-69). Laval : Presses Universitaires.

Félix, C., Amigues R., Espinassy, L., (2014). Observer le travail enseignant. In NumaBocage, L., Marcel, J-F, Chaussecourte, P. (Eds), De l'observation des pratiques enseignantes (pp .52-62). Recherches en Education N¹9, Nantes : CREN.

Félix, C., Saujat, F. (2008). L'aide au travail personnel des élèves entre déficit de prescriptions et savoirs méthodologiques : un double regard didactique et ergonomique. Les Dossiers des Sciences de l'Education, 20, 123-136.

Félix, C., Saujat, F., \& Combes, C. (2012). Des élèves en difficulté aux dispositifs d'aide: 


\section{eJRIEPS Numéro Spécial n¹ Avril 2018}

une nouvelle organisation du travail enseignant. Nouveaux c@hiers de la Recherche en Education / Recherche en Education, (Hors-Série n4), 19- 30.

Johsua, S, Félix, C. (2002). Le travail des élèves à la maison : une analyse didactique en termes de milieu pour l'étude. Revue française de pédagogie, vol. 141, Vers une didactique comparée, 89-97.

Johsua, S., Dupin, J-J. (2001). Introduction à la didactique des sciences et des mathématiques. Paris : PUF.

Leblanc, S., Ria, L., Veyrunes, P. (2013). Vidéo et analyse in situ des situations d'enseignement et de formation dans le programme du cours d'action. In Veillard, L. \& Tiberghien, A. Instrumentation de la recherche en Education. Le cas du développement d'une base de vidéos de situation d'enseignement et d'apprentissage ViSA (pp.63-94). Maison des Sciences de l'Homme.

Leontiev, A.N. (1984). Activité, conscience, personnalité. Moscou : Éditions du Progrès.

Mayen, P. (2012). Les situations professionnelles : un point de vue de didactique professionnelle. Phronesis, vol.1, p.59-67. http://id.erudit.org/iderudit/1006484ar

Mayen, P. (1999). Des situations potentielles de développement. Éducation Permanente, 139, 65-86.

Oddone, I., Re, A., Briante, G. (1981). Redécouvrir l'expérience ouvrière. Vers une autre psychologie du travail, Paris : éditions sociales.

Pastré, P. (2011). La didactique professionnelle, approche anthropologique du développement chez les adultes. Paris, PUF.

Regnard, F. \& Cramer, E. (2003). Apprendre et enseigner la musique : représentations croisées. Paris : L'Harmattan.

Sensevy, G. (2007). Des catégories pour décrire et comprendre l'action didactique. In G. Sensevy \& A. Mercier (dir.), Agir ensemble. L'action didactique conjointe du professeur et des élèves (pp. 13-49). Rennes : Presses Universitaires de Rennes.

Terrien, P. (2011). Quelle recherche pour quel musicien-enseignant ? In Joliat, F. (éd.). Professionnaliser la formation des enseignants de musique (pp. 31-56). Paris: L'Harmattan.

Terrien, P. (2011). La recherche dans la formation des professeurs d'enseignement spécialisés. In Leroy, J.-L., Terrien, P. (éd.). Perspectives actuelles de la recherche en éducation musicale (pp. 165-173). Paris : L'Harmattan.

Terrien, P. (2015). Une petite histoire pédagogique: la prise en main du basson. Réflexions didactiques sur l'enseignement musical (pp. 53-73). Sampzon: 
eJRIEPS Numéro Spécial n¹ Avril 2018

éditions Délateur France.

Theureau, J. (2006). Le cours d'action : méthode développée. Octarès : Toulouse.

Vérillon, P. (2005). Processus productifs et constructifs dans les activités physiques et sportives : la place de l'instrument. Impulsions, 4, 305-325.

Vygotski, LS. (1925/2005). Psychologie de l'art. Paris : La Dispute.

Wallon, H. (1954/1969). "Les milieux, les groupes et la psychogenèse de l'enfant ». Enfance, numéro spécial, p. 287-296.

Wisner, A. (1995). Réflexions sur l'ergonomie (1962-1995). Toulouse : Octarès.

\section{Textes administratifs}

Schéma national d'orientation pédagogique (2008). Ministère de la Culture et de la Communication. Ministère de l'Education Nationale, de l'Enseignement Supérieur et de la Recherche :

Loi n $2013-595$ du 8 juillet 2013 d'orientation et de programmation pour la refondation de l'école de la République.

https://www.legifrance.gouv.fr/affichTexte.do;jsessionid=?cidTexte=JORFTEXT00002767

7984\&dateTexte=\&oldAction=rechJO\&categorieLien=id

Réforme du collège Journal Officiel du 20/05/2015. Décret relatif à l'organisation des enseignements au collège.

https://www.legifrance.gouv.fr/affichTexte.do?cidTexte=JORFTEXT000030613326\&categ orieLien=id

Nouveaux programmes d'enseignement de 2015 (B.O. Spécial №11, du 26/11/2015)

http://www.education.gouv.fr/pid285/bulletin officiel.html?pid bo $=33400$

Education Artistique et Culturelle (B.O. N²8, 09/07/2015)

http://www.education.gouv.fr/pid25535/bulletin officiel.html?cid bo=91164 